\title{
Osmotic stress- and indole-3-butyric acid-induced NO generation are partially distinct processes in root growth and development in Pisum sativum
}

\author{
Zsuzsanna Kolbert*, Bernadett Bartha and László Erdei \\ Department of Plant Biology, Faculty of Science and Informatics, University of Szeged, PO Box 654, 6701 Szeged, Hungary
}

\author{
Correspondence \\ *Corresponding author, \\ e-mail: kolzsu@bio.u-szeged.hu \\ Received 15 November 2007; revised 18 \\ December 2007 \\ doi: 10.1111/j.1399-3054.2008.01056.x
}

In this work, the effects of osmotic stress and exogenous auxin (indole-3butyric acid, IBA) on root morphology and nitric oxide (NO) generation in roots were compared in pea plants. Five-day-old plants were treated with $0,10^{-3}$, $10^{-4}, 10^{-5}, 10^{-6}, 10^{-7}, 10^{-8}$ or $10^{-9} \mathrm{M}$ IBA or with PEG 6000 at concentrations that determined 0,50, 100, 200 or 400 mOsm in the medium, during 5 days. NO generation was examined by in situ and in vivo fluorescence method. Increasing concentrations of PEG as well as IBA resulted in shortening of primary root (PR), enhancement of lateral root (LR) number and significant increase of NO generation. Time-dependent investigations revealed that in the case of IBA treatments, the LR number increased in parallel with an intensified $\mathrm{NO}$ generation, while elongation of PR was not followed by changes in NO levels. Under osmotic stress, the time curve of NO development was distinct compared with that of IBA-treated roots, because significantly, the appearance of lateral initials was preceded by a transient burst of NO. This early phase of NO generation under osmotic stress was clearly distinguishable from that which accompanied LR initiation. It is concluded that osmotic stress and the presence of exogenous auxin resulted in partly similar root architecture but different time courses of $\mathrm{NO}$ synthesis. We suppose that the early phase of $\mathrm{NO}$ generation may fulfill a role in the osmotic stressinduced signalization process leading to the modification of root morphology.

\section{Introduction}

Development of root architecture greatly depends on both environmental and endogenous factors. Water and nutrient availability (Malamy and Ryan 2001, Zhang et al. 1999) as well as interplay between plant hormones like auxin, cytokinins and signaling substances, e.g. nitric oxide (NO), determine the growth of PR and initiation of LR (Correa-Aragunde et al. 2004, Kolbert et al. 2005, 2008, Malamy and Benfey 1997). Lateral roots (LR) are formed from specially positioned cells in the root pericycle postembryonically, which circumstance pro- vides broad phenotypic plasticity during further growth and development. The frequency of initiation of LRs is, in part, determined by the auxin concentration: exogenous application of auxin increases the number of LR. The level of auxin, however, is the function of its basipetal polar transport to the shoot base followed by the acropetal movement to the root apex, and again, basipetal movement toward the shoot-root junction (Casimiro et al. 2001, Swarup et al. 2007) as well as its metabolism. Recently, it was shown that indole-3-butyric acid (IBA), a naturally occurring auxin besides the major IAA, is

Abbreviations - cPTIO, 2-(4-carboxyphenyl)-4,4,5,5-tetramethylimidazoline-1-oxyl-3-oxid potassium salt; DAF-2DA, 4,5diaminofluorescein diacetate; IBA, indole-3-butyric acid; LR, lateral root; $\mathrm{NO}$, nitric oxide; $\mathrm{NO}^{+}$, nitrosonium cation; $\mathrm{NO}^{-}$, nitroxyl anion; NO', nitric oxide radical; NPA, naphtylphtalamic acid; PR, primary root. 
involved in root formation especially in case of adventitious rooting from stem tissue (Ludwig-Müller et al. 2005). Interestingly, the activity of IBA synthetase is enhanced by osmotic stress administered either as drought, sorbitol or $\mathrm{NaCl}$ as well as by $\mathrm{ABA}$, jasmonic acid and salicylic acid, hormones that are involved in stress responses (Ludwig-Müller et al. 1995). The rib1 (resistant to IBA) mutant of Arabidopsis is characterized by short primary root $(\mathrm{PR})$ and increased number of $L R s$ as well as variable responses to environmental stimuli; in this way in its responses, the mutant is separable from the IAA responsive phenotypes, suggesting IBA as a true endogenous auxin in Arabidopsis (Poupart and Waddell 2000). This was confirmed by the fact that IAA and IBA use different auxin transport protein complexes, distribute differently in the plant and the transport of IBA is resistant to the IAA transport inhibitor naphtylphtalamic acid (NPA) (Rashotte et al. 2003). Whether or not IBA is acting directly or serves as substrate for its conversion to IAA (Chhun et al. 2004, Kaldorf and Ludwig-Müller 2000, Zolman et al. 2000), this interrelationship between endogenous and environmental factors offers a genuine model for the study of the mechanism of root architecture development as influenced by external factors.

The molecular, biochemical and physiological events participating between signal transduction and root initiation and development, are, however, less understood. In the last years NO has emerged as a multifunctional bioactive molecule in plant signal transduction pathways participating in a broad spectrum of physiological and developmental processes via direct or indirect processes or through cross talking with the classical plant hormones (review; Lamattina et al. 2003). Root formation is not exception from the targets of NO action (Correa-Aragunde et al. 2004, 2006, Kolbert et al. 2005).

$\mathrm{NO}$ is a free radical diffusible, lipophylic gas which has three forms in both animals and plants: $\mathrm{NO}^{+}$(nitrosonium cation), $\mathrm{NO}^{-}$(nitroxyl anion) and $\mathrm{NO}^{\circ}$ (nitric oxide radical). Because of its lipophylic characteristics, it can diffuse through membranes. NO has major role in PR growth and LR formation because it acts as a signal molecule in auxin-induced signal transduction. Auxin induces NO production in an unknown way, which activates guanylate cyclase enzyme and cGMP is produced (Pagnussat et al. 2003), which is in the route toward activation of cell division and differentiation.

Our previous experiments show that NO is an active signal component in different stress responses like drought and heavy metal load. Interestingly, the appearance of NO, as the function of time and spatial localization in roots, suggests different mechanisms involved in $\mathrm{NO}$ production and in the role of signal transduction (Bartha et al. 2005, Kolbert et al. 2005).

Recently, it was suggested that stress-induced morphogenetic responses are controlled by auxin distribution in the plant (Potters et al. 2007). The question arose if there are similarities or differences in growth responses of a root system under external auxin load and osmotic stress, with focus on the role of $\mathrm{NO}$ in the phenotypic acclimation processes. In this work, the production of $\mathrm{NO}$ is investigated in the processes of IBA-induced LR initiation and PR elongation as compared with those under osmotic stress conditions. Using IBA and PEG as osmotic agent in broad concentration range during the time period of stress response as well as NO scavenger agent, different roles of NO could be distinguished under these conditions.

\section{Materials and methods}

\section{Plant material and growth conditions}

Pea (Pisum sativum L. cv. Rajnai törpe $=$ Petit Provençal) seeds were surface sterilized with $5 \%$ sodium hypochlorite for $10 \mathrm{~min}$, rinsed and imbibed for $2 \mathrm{~h}$ in running water. Germination took place between moisture filter papers in Petri dishes at $26^{\circ} \mathrm{C}$ for 2 days. Germs with 2$\mathrm{cm}$-long radicles were then placed into modified Hoagland solution (nine seedlings per $400 \mathrm{ml}$ growth vessels) and were grown under controlled conditions in greenhouse at photo flux density of $240 \mu \mathrm{mol} \mathrm{m} \mathrm{m}^{-2} \mathrm{~s}^{-1}$ (12/ $12 \mathrm{~h}$ day/night period) at relative humidity of $55-60 \%$, and $25 \pm 2^{\circ} \mathrm{C}$ temperature for 5 days. The modified Hoagland solution contained the following chemicals: $2 \mathrm{mM} \mathrm{Ca}\left(\mathrm{NO}_{3}\right)_{2}, 1 \mathrm{mM} \mathrm{MgSO}, 0.5 \mathrm{mM} \mathrm{KCl}, 0.5 \mathrm{mM}$ $\mathrm{KH}_{2} \mathrm{PO}_{4}$ and $0.5 \mathrm{mM} \mathrm{Na} 2 \mathrm{HPO}_{4}$. The micronutrient concentrations used for preparation of the nutrient solutions were: $1 \mu \mathrm{M} \mathrm{MnSO}_{4}, 5 \mu \mathrm{M} \mathrm{ZnSO}_{4}, 0.1 \mu \mathrm{M}$ $\left(\mathrm{NH}_{4}\right)_{6} \mathrm{MO}_{7} \mathrm{O}_{24}, 10 \mu \mathrm{M} \mathrm{H} \mathrm{BO}_{4}, 0.1 \mu \mathrm{M} \mathrm{AlCl}_{3}$, and $20 \mu M$ Fe-EDTA. Five-day-old plants were treated for another 5 days. Sampling was taken daily or as stated. The PR length was recorded in $\mathrm{mm}$ using a scale. The LR number was counted and expressed as initials per root.

\section{Chemicals}

NO was detected with the help of a fluorescent dye, 4,5diaminofluorescein diacetate (DAF-2DA; Sigma-Aldrich, St Louis, MO) dissolved in MES buffer (MES and $\mathrm{KCl}, \mathrm{pH}$ 6.15). MES and $\mathrm{KCl}$ was obtained from Reanal (Budapest, Hungary). For inhibiting the polar transport of auxin, NPA (Sigma-Aldrich) was used. For scavenging NO, 2-(4carboxyphenyl)-4,4,5,5-tetramethylimidazoline-1-oxyl3-oxid potassium salt (cPTIO; Sigma-Aldrich), and as exogenous auxin, IBA (Fluka, Seelze, Germany) were used. 


\section{Treatments}

Osmotic stress was administrated by dissolving PEG 6000 (Sigma) into the nutrient solution at 50, 100, 200 and 400 mOsm osmotic concentration as measured by a digital automatic osmometer (Micro GMS, Hungary). These concentrations are equivalent to 6.98, 9.97, 14.3 and $19 \%(\mathrm{w} / \mathrm{v})$, or $-0.122,-0.244,-0.488$ and -0.976 MPa $\psi_{\mathrm{s}}$ as calculated according to Wyn Jones and Gorham (1983), respectively. For auxin treatment, $10^{-3}$ $10^{-9} \mathrm{M}$ indole-3-butyric-acid (IBA) concentrations were applied. As for verification of $\mathrm{NO}$ detection, the $\mathrm{NO}$ scavenger cPTIO was used in $200 \mu \mathrm{M}$ concentration. As polar auxin transport inhibitor, NPA was applied at $10 \mu \mathrm{M}$ concentration.

\section{Detection of NO}

Visualization of NO was performed as the highly sensitive in situ and in vivo method of Kojima et al. (1998) as applied for plant tissues (Pedroso et al. 2000). Root segments of 1.5-2 cm length and cut cross-sections were dyed with $10 \mu \mathrm{M}$ DAF-2DA dissolved in MES/KCI buffer for $20 \mathrm{~min}$ at $25 \pm 2{ }^{\circ} \mathrm{C}$ in darkness. Cross-sections were cut with a specific plant microtome (NIHONIKA MTH-1, Tokyo, Japan). After dyeing the samples were washed $4 \times$ within 20 min with MES buffer ( $1 \mathrm{mM}$, pH 6.15) and were prepared on microscopic slides.

To detect fluorescence intensity Zeiss Axiowert 200Mtype fluorescent microscope (Carl Zeiss, Jena, Germany) connected with a high resolution digital camera (Axiocam HR) was used. FLUAR 5×/0.25 NA and FLUAR $10 \times / 0.50$ NA objective lenses were used to investigate the samples.
Digital photographs were taken from the samples with a high-resolution digital camera (Axiocam HR, HQ CCD camera; Carl Zeiss). To measure the fluorescence intensity, AxIOvisıon Rel. 4.5 software was applied using a filter set 10 . The fluorescent values were relative to the area of the sample analyzed. The same camera settings were recorded for each digital image. The detection of $\mathrm{NO}$ fluorescence was performed at 0, 24, 48, 72, 96 and $120 \mathrm{~h}$ after treatment of roots, and in some cases also at 12 and $36 \mathrm{~h}$ (noted at those sites). In each treatment, at least three samples were measured. Three independent series of plants as biological repetitions were grown $(n=9)$. The selected fluorescent images are representatives of similar results from the three repetitions.

\section{Statistics}

The data presented as mean \pm SE were obtained from at least three independently grown series of plants. Significant differences were determined using the Student's $t$-test applying SigmaStat 3.11 software. Significant differences among means $(\mathrm{n}=9)$ are indicated by one $(P \leq 0.05)$, two $(P \leq 0.01)$, or three $(P \leq 0.001)$ asterisk(s).

\section{Results}

\section{The effects of osmotic stress and IBA treatment on root morphology and NO production are similar but not identical}

Increasing osmolarities of PEG in the growth solution enhanced the number of LR while decreased the lengths of PR of pea plants (Fig. 1A). The concomitant NO
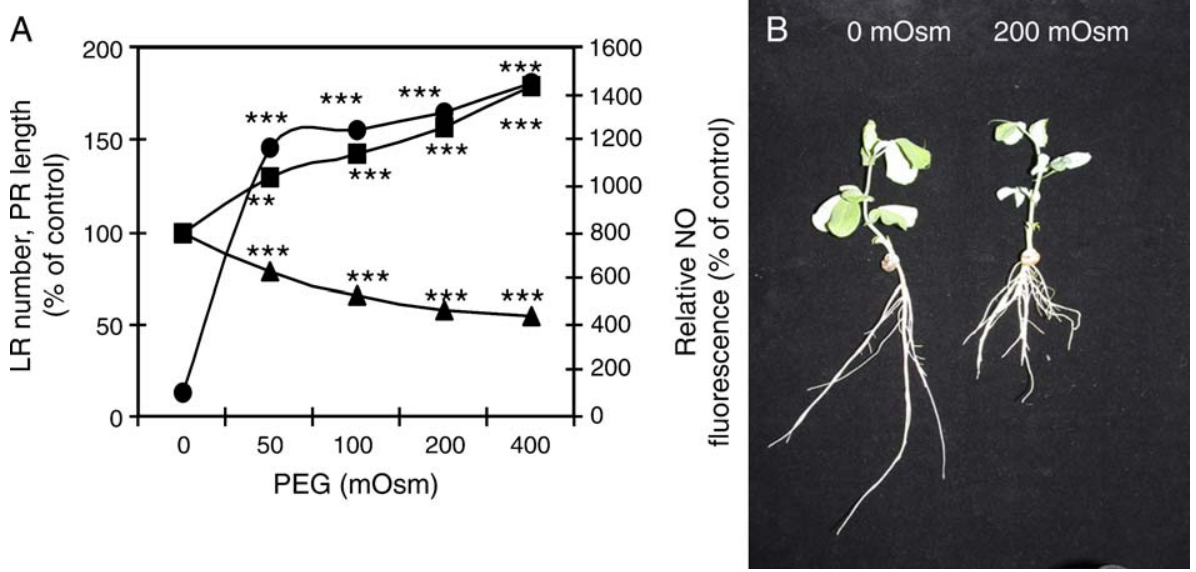

Fig. 1. (A) Effects of PEG-induced osmotic stress on the growth of PR ( $\boldsymbol{\Delta})$, development of $L R(\boldsymbol{\square})$ and NO formation in the roots ( $\boldsymbol{O})$ of Pisum sativum $L$. Data are expressed as $\%$ of control as follow: for PR, $100 \%=154 \pm 15 \mathrm{~mm} \mathrm{plant}^{-1}$, for LR, $100 \%=14 \pm 2$ pieces plant $^{-1}$ and for $\mathrm{NO}$, $100 \%=906 \pm 250$ pixel intensity. Each point is the mean \pm SE of nine replicates per treatment $(n=9)$ and statistical differences are indicated by ${ }^{*} P \leq 0.05,{ }^{*} P \leq 0.01$ and $* * * P \leq 0.001$. B) Morphological changes after PEG-treatment as compared with control plants. 
fluorescence curve (Fig. 1A), after an intensive initial burst in response to the lowest osmotic value applied, followed the increase of numbers of LR initials in a parallel way. The phenotypic acclimation resulted in a strongly modified morphology of the plant, especially root architecture (Fig. 1B). This phenomenon is comparable with that which appeared after auxin treatments (Fig. 2). In agreement with earlier observations, we found that low IBA concentrations $\left(10^{-9}-10^{-8} \mathrm{M}\right)$ resulted in longer PR and fewer LR compared with those of control plants, while higher IBA concentrations $\left(10^{-7}-10^{-3} \mathrm{M}\right)$ caused shorter PR and enhanced LR number/root values. Concerning NO production, low (control) level NO fluorescence was detected in the cases of low auxin treatments; however, in roots treated with $10^{-6}$ and $10^{-5} \mathrm{M} \mathrm{IBA}$, the intensity of $\mathrm{NO}$ fluorescence was two to three times higher than in the control plants. In the higher concentration range of IBA, the elongation of PRs was inhibited (Fig. 2B). The morphological alterations are shown in Fig. 2B. NO fluorescence for the low- and high-concentration region is represented by the photographs taken from cross sections of roots grown at 0,50 and 400 mOsm PEG, and $0,10^{-8}$ and $10^{-5}$ MIBA (Fig. 3A-C and D-F, respectively).

A very simple but informative experiment was carried out to investigate the relationship between exogenous auxin treatment and $\mathrm{NO}$ generation within pea roots. Three groups of 5-day-old plants were used: (1) plants treated for 10 days with $10^{-8}$ or $10^{-5} \mathrm{M} \mathrm{IBA}$; (2) plants treated with $10^{-8} \mathrm{MIBA}$ for 5 days and then transferred into solution containing $10^{-5} \mathrm{M}$ IBA and (3) plants treated with $10^{-5} \mathrm{M}$ IBA for 5 days then transferred into a solution containing $10^{-8} \mathrm{MIBA}$. Samples were taken at the 5th and the 10th day of treatments. The short laterals that appeared after 5-day-long $10^{-5} \mathrm{M}$ IBA treatment were elongated during the following 5 days (Fig. 4f); however, in the case of plants transferred to the lower auxin concentration $\left(10^{-8} \mathrm{M} I \mathrm{IBA}\right)$, the elongation of laterals were more intensive (Fig. 4h). Importantly, along this developmental transient, the intensive NO fluorescence, which was earlier generated in the presence of $10^{-5} \mathrm{M}$ IBA after 5 days, strongly decreased when the plants were transferred into $10^{-8} \mathrm{MIBA}$ (Fig. $4 \mathrm{H}$ ), while only slight decrease was detected in the roots staying in $10^{-5} \mathrm{M}$ IBA for 10 days (Fig. 4F). Plants, with roots elongated in $10^{-8} \mathrm{M}$ IBA during the first 5 days, developed short laterals after transferring them into higher IBA concentration and showed increased NO fluorescence (Fig. 4g,G). These results provide evidence for possible relationship between auxin level and NO generation of pea roots.

In our experiments, the application of the scavenger CPTIO, in agreement with Correa-Aragunde et al. (2004), clearly demonstrated that NO plays a role in LR formation because it completely inhibited LR formation both alone or together with $10^{-5} \mathrm{MIBA}$, but had no effect on PR elongation (data not shown).

Under osmotic treatments, root development was similar to that of IBA-treated plants as shown in Figs 1 and 2 , respectively. The question arose if under osmotic

A
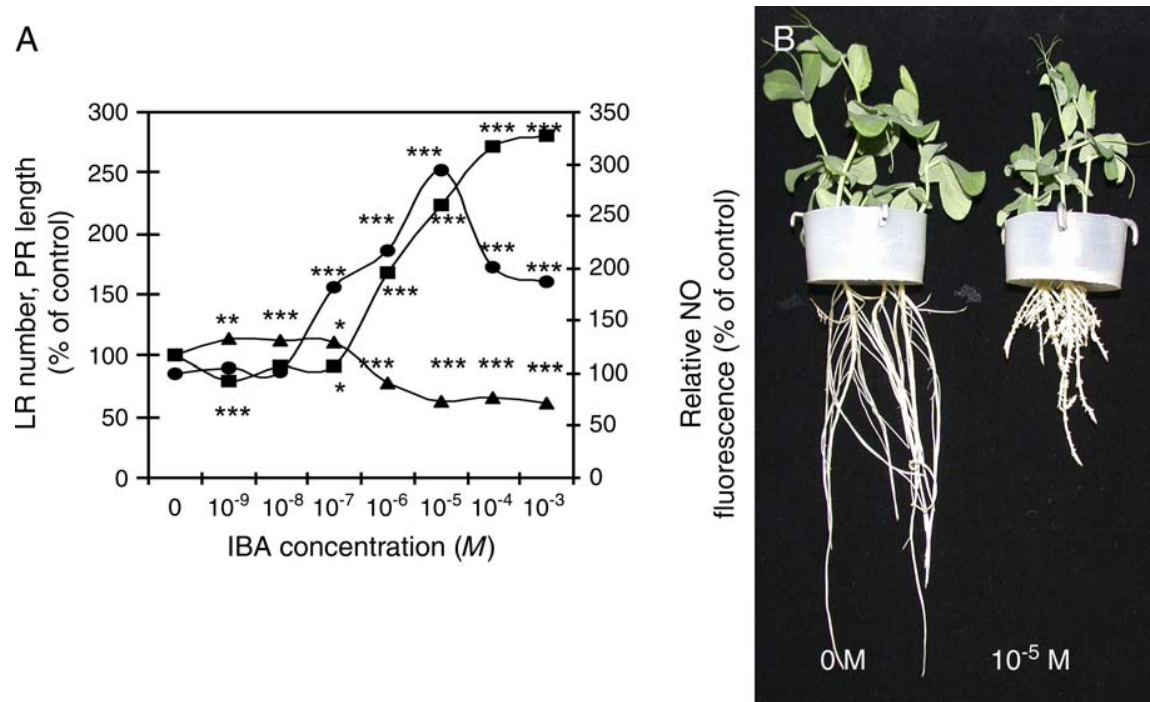

Fig. 2. (A) Effects of IBA concentrations on the growth of PR ( $\mathbf{\Delta})$, development of $L R(\boldsymbol{\square})$ and NO formation in the roots (O) of Pisum sativum L. Data are expressed as $\%$ of control as follow: for PR, $100 \%=144 \pm 24 \mathrm{~mm} \mathrm{plant}^{-1}$, for LR, $100 \%=25 \pm 3$ pieces plant $^{-1}$ and for $\mathrm{NO}, 100 \%=1120 \pm 126$ pixel intensity. Each point is the mean \pm SE of nine replicates per treatment $(\mathrm{n}=9)$ and statistical differences are indicated by * $P \leq 0.05, * * P \leq 0.01$ and $\star * * P \leq 0.001$. (B) Morphological changes after $10^{-5} \mathrm{M}$ IBA treatment as compared with control plants. 

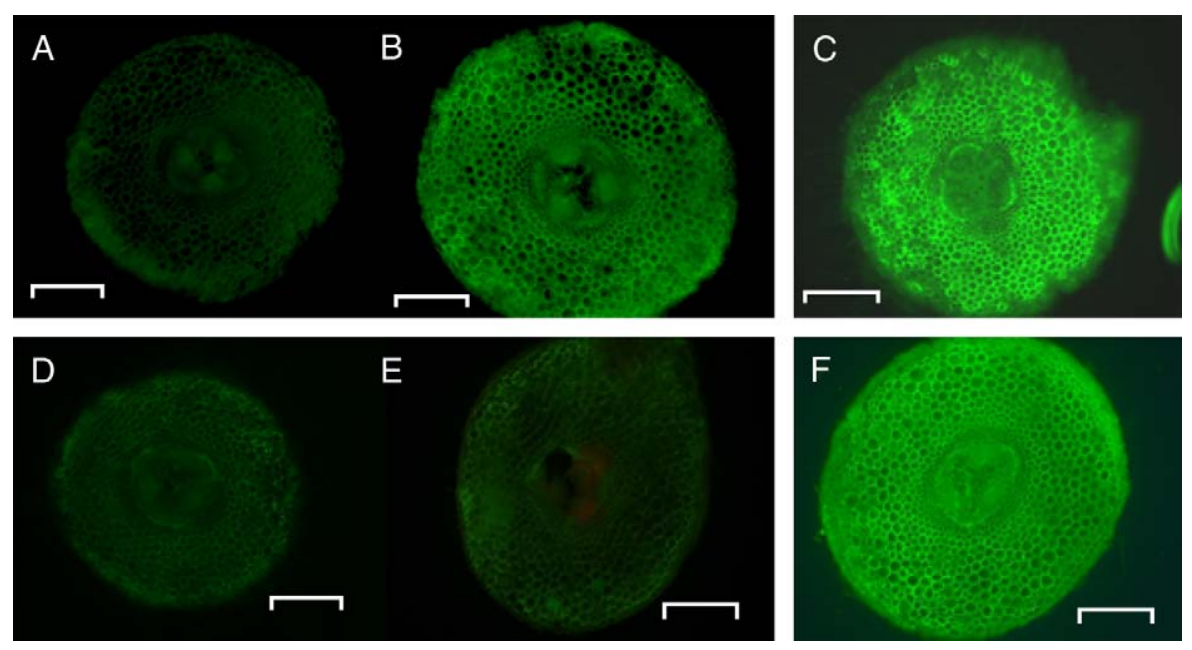

Fig. 3. Fluorescence visualization of $N O$ production in cross sections of pea PRs in the control ( $0 \mathrm{mOsm} P E G, A ; 0 M I B A, D), 50 \mathrm{mOsm} P E G(B)$, 400 mOsm PEG (C), $10^{-8} \mathrm{MIBA}-(\mathrm{E})$ and $10^{-5} \mathrm{M}$ IBA- $(\mathrm{F})$ treated samples. Representative images for the concerned concentrations in the NO curve are shown in Fig. 2a. The selected images represent the mean values of nine replicates per treatment. Root samples were prepared and investigated as described in Materials and methods. Bars $=500 \mu \mathrm{m}$.

stress acropetally transported auxin was responsible for the intensified formation of LR and NO synthesis. Using the auxin polar transport inhibitor, NPA, the number of LR initials and the NO level were decreased, while the growth of PR practically was not affected (Fig. 5A-C). The relative low inhibition of LR development by NPA can be explained by the fact that NPA is not an inhibitor of transport of IBA (Rashotte et al. 2003) but it indicates that at least for a low extent, IAA can also be responsible for LR formation. The inhibition of NO generation was more significant under osmotic conditions, which could be because of the different time curves and levels of $\mathrm{NO}$ generation during the two treatments.

\section{Osmotic stress- and IBA-induced NO generations have different time curves: 'the stress-NO'}

In time-dependent investigations, the length of PR, number of LR and fluorescence of $\mathrm{NO}$ were measured at the $0,24,48,72,96$ and 120th h of treatments. These experiments were carried out in all PEG and IBA concentrations, but for simplicity we chose two representative concentrations, namely 50 and $400 \mathrm{mOsm}$ PEG, and $10^{-8}$ and $10^{-5} \mathrm{MIBA}$. For the $\mathrm{NO}$ production under osmotic stress, NO determination was carried out additionally also at 12 and $36 \mathrm{~h}$.

As the function of time, constant linear growth of PR was observed in control roots and only the slopes not the linearity of the curves were changed by osmotic or IBA treatments (Fig. 6A, D). The length of PR was decreased by both concentrations of PEG (Fig. 6A). However, elongation was significantly enhanced by $10^{-8} \mathrm{M}$ IBA, while treatment with $10^{-5} \mathrm{M}$ IBA resulted in immediate cease of PR elongation (Fig. 6D).

Initiation of LR development was observed after $48 \mathrm{~h}$ of osmotic treatment, showing proportionally increasing number of LR with increasing osmotic concentration (Fig. 6B). Concerning IBA treatments, enhancement of LR number was equal in time in the case of control and $10^{-8}$ M IBA-treated pea plants. Actually, the latter group of plants had much fewer LRs/root than the control. Until the 48th $\mathrm{h}$ of treatment, IBA at $10^{-5} \mathrm{M}$ exhibited no significant effect on the visible appearance of LR number, but after this lag phase the number of LRs increased significantly and the most intensive enhancement was observed between the 48th and 72th $\mathrm{h}$ of treatment (Fig. 6E). In this respect, the effects of osmotic stress and high concentration of external auxin when compared with the time courses in Fig. 6B, E, were apparently identical.

There is, however, significant difference in the time courses of NO production under osmotic stress and IBA treatments. Under osmotic stress, a fast increase in $\mathrm{NO}$ level appeared reaching plateau between 24 and $36 \mathrm{~h}$ followed by steady increase afterwards (Fig. 6C).

Control and $10^{-8} \mathrm{M}$ IBA-treated roots showed similar low levels of NO fluorescence intensity which increased slightly in time. In the case of $10^{-5} \mathrm{M}$ IBA, visible fluorescence increase could be detected only after the 48th $\mathrm{h}$ of treatment (Fig. 6F). Comparison of time courses of $\mathrm{NO}$ production in Fig. 6C, F strongly suggest the occurrence of a transient $\mathrm{NO}$ burst that precedes the slow and steady auxin-coupled $\mathrm{NO}$ generation. 

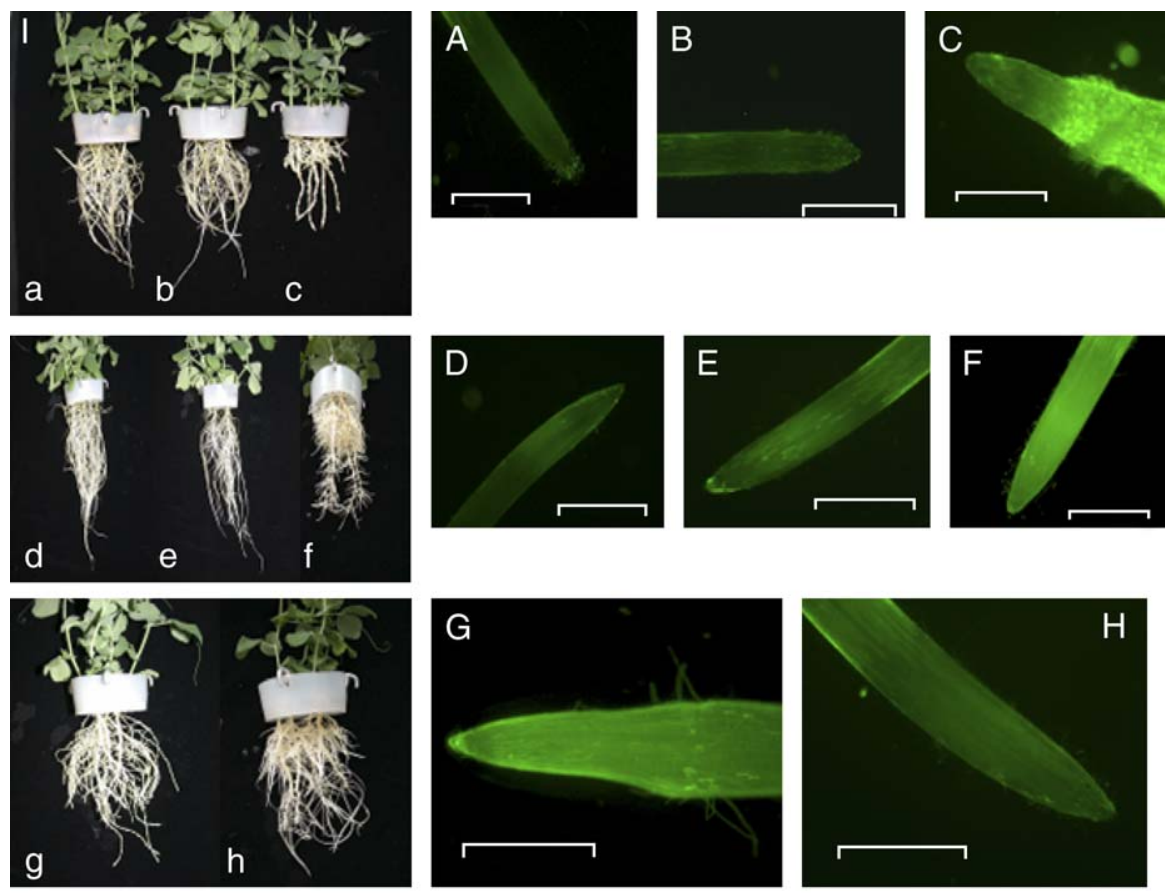

II

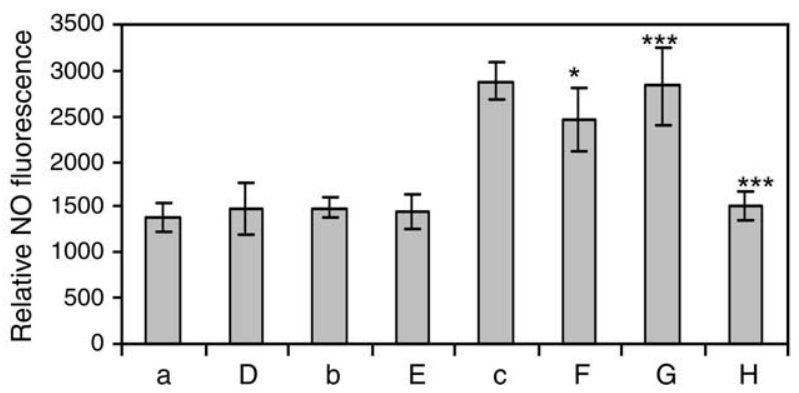

Fig. 4. ' $I$ ', Root morphology (a-h) and NO fluorescence (A-H) of pea plants after 5 days treatment (control: $\left.a, A ; 10^{-8} M I B A: b, B ; 10^{-5} M I B A: C, C\right)$ and after 10 days treatment (control: d,D; $10^{-8} \mathrm{MIBA}: \mathrm{e}, \mathrm{E} ; 10^{-5} \mathrm{MIBA}$ : f,F). Pictures $\mathrm{g}$ and $\mathrm{G}$ represent plants transferred from $10^{-5} \mathrm{MIBA}$ into the solution containing $10^{-8} \mathrm{MIBA}$, while h and $\mathrm{H}$ show plants transferred from $10^{-8} \mathrm{MIBA}$ into $10^{-5} \mathrm{MIBA}$. Relative fluorescence values of $\mathrm{NO}$ production for $\mathrm{A}-\mathrm{H}$ are shown in 'Il'. Root samples were prepared and investigated as described in Materials and methods. Bars $=1 \mathrm{~mm}$. The selected images are representative of nine photographs per treatments, and the data in 'Il' are the mean $\pm S E(n=9)$. Statistical differences are indicated by ${ }^{*} P \leq 0.05$, $* * P \leq 0.01$ and $* * * P \leq 0.001$.

To clearly separate the two types of NO, the rate of $\mathrm{NO}$ production is represented as the function of time of treatments (Fig. 7). The differential curves clearly distinguish between the osmotic stress-induced transient and the emergence of $\mathrm{NO}$ generation after $48 \mathrm{~h}$ of treatments.

To answer the question, whether the early NO burst has a role in PEG-induced LR development, we suppressed the PEG-induced transient NO burst with CPTIO in the first $36 \mathrm{~h}$. These samples showed decreased LR number compared with control and 400 mOsm PEGtreated roots in the 120th $\mathrm{h}$ of treatment (Fig. 8). This strongly suggests that NO burst is needed to PEGinduced LR development.

\section{Discussion}

The development of root system architecture, besides intrinsic, mainly hormonal factors and cell cycle regulators (Malamy and Benfey 1997), is greatly influenced by environmental factors, most significantly water stress and nutrient availability (Lynch 1995, Malamy 2005, Malamy and Ryan 2001). In the build-up of the architecture, the number and frequency of $L R$ initiations and root elongation are the main processes determined by 'intrinsic' and 'response' pathways (Malamy 2005). In this study, we investigated the effects of an environmental factor (osmotic stress) and a typically internal factor, auxin, on the elongation of PRs and initiation of secondary (lateral) 

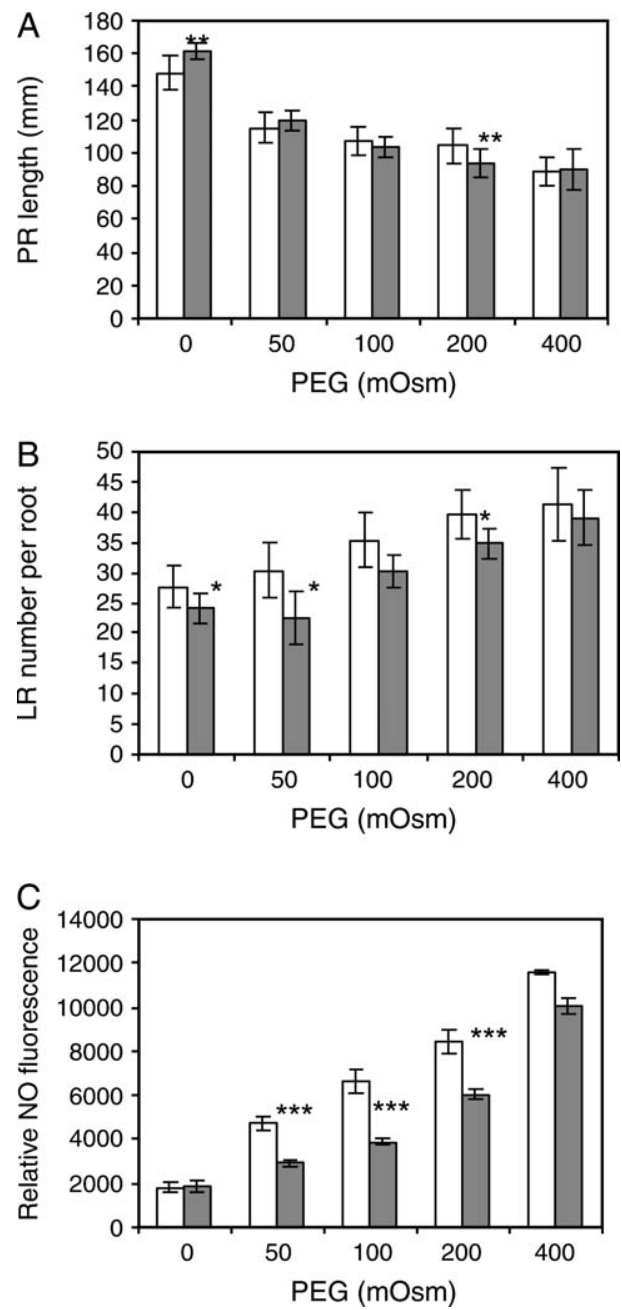

Fig. 5. Effects of PEG concentrations on PR length (A), LR number (B) and NO fluorescence $(C)$ of pea roots. White columns represent treatments without NPA, grey bars represent PEG treatments together with $10 \mu \mathrm{M}$ NPA treatment. Each point is the mean \pm SE of nine replicates per treatment $(\mathrm{n}=9)$ and statistical differences are indicated by $* P \leq 0.05$, $* * P \leq 0.01$ and $* * * P \leq 0.001$.

roots. Although previous studies gave account that $\mathrm{NO}$ mediates the auxin response of LR formation (CorreaAragunde et al. 2004, 2006, Pagnussat et al. 2002), further details and differences were found for the role of $\mathrm{NO}$ in root growth and development under the conditions of water stress and externally added auxin load.

\section{The effects of osmotic stress and IBA treatment on root morphology and NO production are similar but not identical}

Elongation of roots under water deficit is usually strongly hindered; while mild osmotic stress can stimulate growth (van der Weele et al. 2000). Recently, Pasternak et al.
(2005) suggested that key events in plant growth responses under environmental stress are interactions between the oxidative stress and auxin that result in altered auxin transport and hence, modified growth and development. In our experiments, the similarity between responses of PR elongation and $L R$ initiation to increasing water stress and external auxin concentrations support this idea (Figs 1 and 2). In both cases, the reduction of PR length reached approximately $50 \%$ of the control, and the increase of numbers of LRs were $180 \%$ under osmotic stress and about $280 \%$ at the highest IBA concentration. These figures resulted in similar root architecture with slight differences in the length of $L R$ (Figs 1B and 2B). Concerning the increase of LRs under osmotic stress in pea, it has to be mentioned that contrasting result was obtained for Arabidopsis, where the number of LRs decreased significantly and continuously from -0.49 through -0.92 to $-1.21 \mathrm{MPa}$ (van der Weele et al. 2000). The difference may origin from the different species and, in part, from the growing system (there, nutrient-agar medium with PEG 8000).

Despite the similarities in characteristics of root growth and development under osmotic stress and auxin load, there is a significant difference in the intensity of the accompanying NO generation: while the maximal value is three-fold in the roots with the high auxin load (at $10^{-5} \mathrm{M}$ IBA), osmotic stress response resulted in 14-fold increase of NO level at 400 mOsm ( $-0.98 \mathrm{MPa})$ PEG 6000 concentration. Interestingly, the intensive increase of NO production started with and accompanied the LR initiation process in both cases.

The effects of IBA on PR growth and LR development, in connection with NO production, allowed to distinguish clearly between the two processes. It is well known that auxin at low concentration promotes PR elongation, while above a threshold concentration it induces LR initiation (Casimiro et al. 2001, Hinchee and Rost 1986, Torrey 1950). Still, in our actual experimental design, we applied a wide concentration range $\left(10^{-9}-10^{-3} \mathrm{M}\right)$ of exogenous auxin, IBA. Although IBA is mostly used to investigate adventitious rooting, we choose this auxin because of its higher stability than IAA and because it is found naturally in pea roots (Schneider et al. 1985). This wide concentration range of exogenous IBA treatment revealed the two-fold effect on root growth and development characteristics and NO fluorescence as well: low concentrations of IBA $\left(10^{-9}-10^{-8} \mathrm{M}\right)$ was favorable to PR elongation and this process was not followed by $\mathrm{NO}$ generation, while higher IBA concentrations $\left(10^{-7}\right.$ $10^{-3} \mathrm{M}$ ) were responsible for $L R$ emergence and $\mathrm{NO}$ generation. The enhancement of LR number was continual but fluorescence of $\mathrm{NO}$ showed a maximum value at $10^{-5} \mathrm{M}$ IBA (Fig. 2). Fig. 3 illustrates the difference in localization and intensities of $\mathrm{NO}$ generation under 

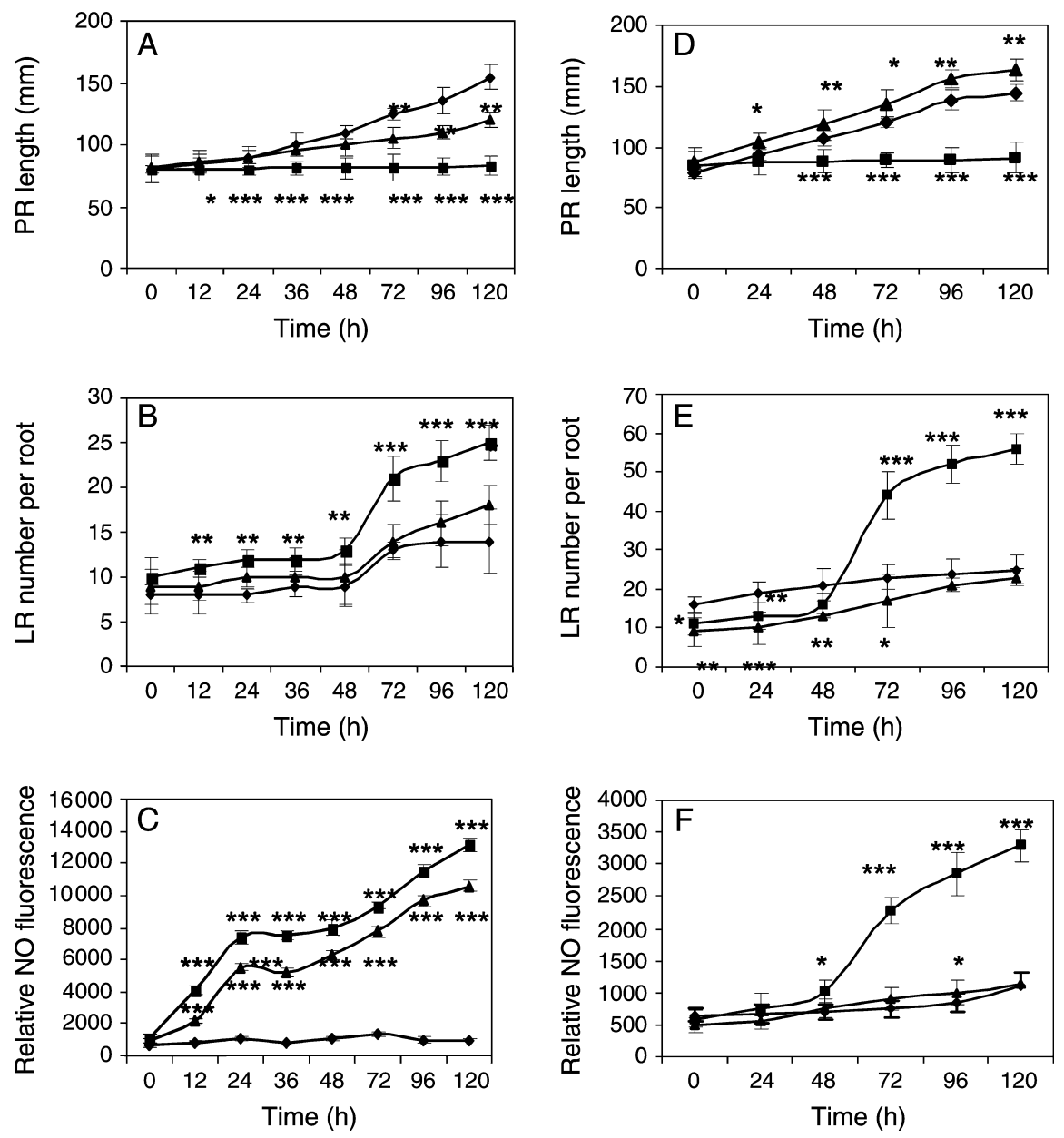

Fig. 6. Time dependence of PR growth (A), LR development (B) and NO formation (C) in control plants ( $)$, 50 mOsm PEG-treated plants $(\boldsymbol{\Delta}), 400 \mathrm{mOsm}$ PEG-treated plants ( $(\mathbf{})$. Time dependence of PR growth (D), LR development (E) and NO formation (F) in control plants $(\bullet), 10^{-8} \mathrm{MIBA}$-treated plants ( $\mathbf{\Delta}$ ), $10^{-5}$ M IBA-treated plants $(\mathbf{\square})$. The data represent the mean values of nine replicates per treatment and the vertical bars are standard errors and statistical differences are indicated by $* P \leq 0.05, * * P \leq 0.01$ and $* * * P \leq 0.001$

osmotic and auxin treatments. That the intensive NO signal is strongly coupled to initiation and emergence of LRs is proved its decay after transferring the plants to the medium of Iow IBA concentration that induced the elongation of the short primordial (Fig. 4, I and II). The first step of LR initiation is the auxin-mediated cell cycle activation (Himanen et al. 2002), whose process, as demonstrated in alfalfa cell cultures, is promoted by $\mathrm{NO}$ (Ötvös et al. 2005). NO fluorescence during LR formation was also observed in tomato roots, as illustrated by Correa-Aragunde et al. $(2004,2006)$.

\section{Osmotic stress- and IBA-induced NO generations have different time curves: the 'stress-NO'}

Investigating the time-dependent properties of rooting processes and NO generation, we observed that the effect of $10^{-5} \mathrm{M}$ IBA on LR number and NO fluorescence appeared after $48 \mathrm{~h}$ treatment. The similar temporal formation of auxin- induced NO synthesis and LR initiation suggests a functional relation between these processes (Fig. 6E, F). This supposition was confirmed by the result that $\mathrm{NO}$ fluorescence is strongly linked to auxin level in roots (Fig. 4).

During osmotic stress, the time dependence of NO development showed significant difference as compared with that of IBA-treated roots because the appearance of lateral initials was preceded by a transient burst of $\mathrm{NO}$ (Fig. 6). This early phase of $\mathrm{NO}$ generation under osmotic stress, culminating at $24 \mathrm{~h}$, was clearly distinguishable from that which accompanied LR initiation under both osmotic and IBA treatments. The source and the role of this $\mathrm{NO}$ transient are certainly different from those of the constant and steady NO phase, which start after $48 \mathrm{~h}$ of 


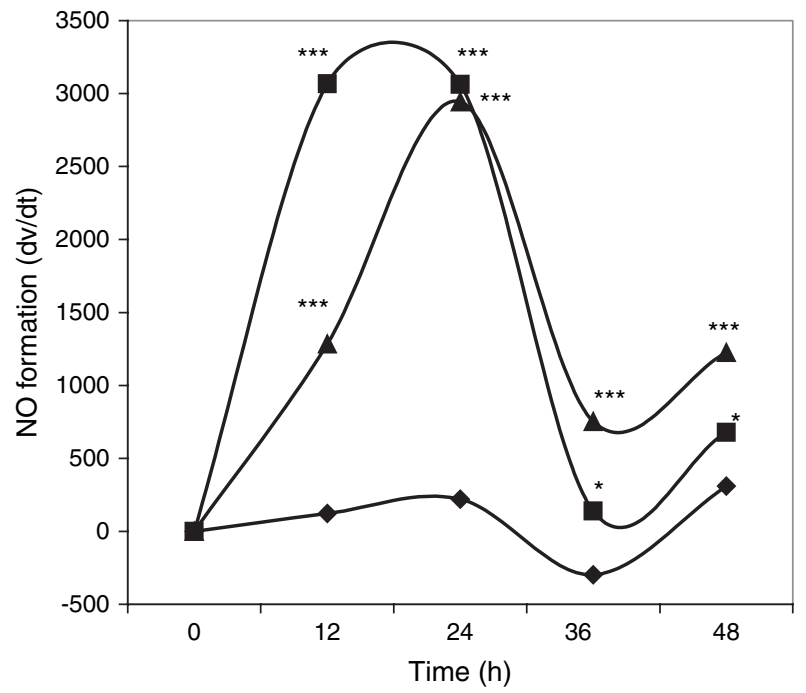

Fig. 7. Differential time curves of transient NO formation in control (•), 50 mOsm PEG-treated ( $(\mathbf{\Lambda})$ and 400 mOsm PEG- treated ( $\mathbf{\square})$ pea roots and statistical differences are indicated by ${ }^{*} P \leq 0.05,{ }^{*} P \leq 0.01$ and $\star * * P \leq 0.001$

the treatments, when the transient one already has decayed. The kinetics of early $\mathrm{NO}$ transients seem to depend on the source of NO. For instance, very early NO burst, developed in $30 \mathrm{~min}$, was observed in response to $\mathrm{Fe}^{2+}$ treatment in Arabidopsis, preceding ATFer1D gene expression (Arnaud et al. 2006), or in the case $\mathrm{Cu}^{2+}$ treatments of Pisum sativum and Brassica juncea, when the $\mathrm{NO}$ peak was obtained after $2 \mathrm{~h}$ after subjecting the plants to the metal ions (Bartha et al. 2005). Both these transition metal ions, in the Fenton reaction with $\mathrm{H}_{2} \mathrm{O}_{2}$, produce hydroxyl radicals leading to the formation other reactive oxygen species (ROS). Oxidative stress may involve $\mathrm{NO}$ as well playing a role in drought responses in the $A B A$ pathway in wheat root (Zhao et al. 2001). A slower early NO burst was obtained in a fungal elicitor-induced process that had a maximal value around $5 \mathrm{~h}$ after treatment, preceding salicylic acid response (Xu et al. 2006), or at $24 \mathrm{~h}$ after the treatment of wheat plants with stripe rust (Guo et al. 2004). When the osmotic stress-induced NO burst was eliminated by the NO-scavenger cPTIO, less LRs were formed than in control and osmotic-stressed roots, indicating that the early NO burst ('stress-NO') is necessary for the osmotic stress-induced LR formation.

These findings refer to the different sources and the localization of $\mathrm{NO}$ in the signal transduction pathway, i.e. it seems that $\mathrm{NO}$ is signaling in the early steps in stress responses, preceding the specification of the different pathways.

Abiotic stressors, thus osmotic effects cause oxidative stress. Plant growth responses under these conditions are modified by altered auxin transport (Pasternak et al. 2005),
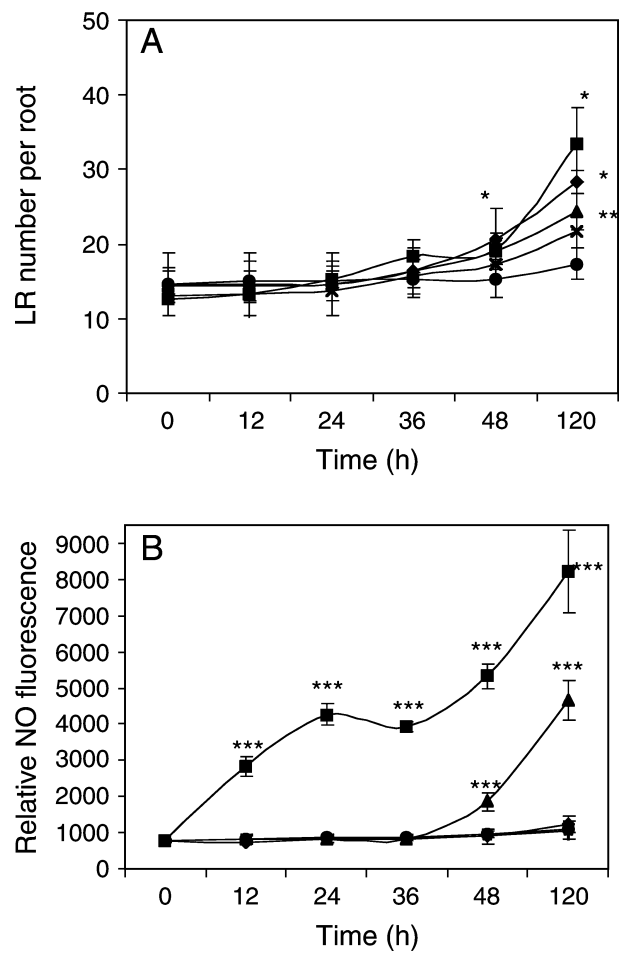

Fig. 8. Time curves of $L R$ development (A) and NO generation (B) of control $(\bullet), 400$ mOsm PEG- $(\mathbf{\bullet})$, CPTIO + PEG- $(\mathbf{x})$, CPTIO- $(\bullet)$ treated pea plants and those of in plants transferred after the 36th hour from CPTIO + PEG into a solution containing 400 mOsm PEG ( $\mathbf{\Lambda})$. The data represent the mean values of nine replicates per treatment and the vertical bars are standard errors. Statistical differences are indicated by ${ }^{*} P \leq 0.05,{ }^{* *} P \leq 0.01$ and ${ }^{* * *} P \leq 0.001$.

hence the similarity, though not identical, of osmotic stress-induced and IBA-induced root structures (Figs 1B and $2 \mathrm{~B}$ ). It was suggested that a stress-induced morphogenetic response, in general, is controlled by auxin distribution in the plant and ROS are part of the signalization between the stress and the morphogenetic response (Potters et al. 2007). In our experiments, the early appearance of the NO transient can be enrolled into the signal transduction pathway towards the auxin-induced changes in root morphology, this process has significance in the phenotypic adaptation under drought conditions.

Acknowledgements - This work was supported by the Hungarian Scientific Research Fund grant no. OTKA T048436 and Phare CBC HU 2003/005.830.01-04 to L. E.

\section{References}

Arnaud N, Murgia I, Boucherez J, Briat J-F, Cellier F, Gaymard F (2006) An iron-induced nitric oxide burst precedes ubiquitin-dependent protein degradation for Arabidopsis AtFer1 ferritin gene expression. J Biol Chem 281: 23579-23588 
Bartha B, Kolbert Zs, Erdei L (2005) Nitric oxide production induced by heavy metals in Brassica juncea L. Czern. and Pisum sativum L. Acta Biol Szeged 49 (1-2): 9-12

Casimiro I, Marchant A, Bhalerao RP, Beeckman T, Dhooge S, Swarup R, Graham N, Inzé D, Sandberg G, Casero PJ, Bennett M (2001) Auxin transport promotes Arabidopsis lateral root initiation. Plant Cell 13: 843-852

Chhun T, Taketa S, Tsurumi S, Ichii M (2004) Different behaviour of indole-3-acetic acid and indole-3-butyric acid in stimulating lateral root development in rice (Oryza sativa L.). Plant Growth Regul 43: 135-143

Correa-Aragunde N, Graziano M, Lamattina L (2004) Nitric oxide plays a central role in determining lateral root development in tomato. Planta 218: 900-905

Correa-Aragunde N, Graziano M, Chevalier C, Lamattina L (2006) Nitric oxide modulates the expression of cell cycle regulatory genes during lateral root formation in tomato.

J Exp Bot 57: 581-588

Guo P, Cao Y, Li Z, Zhao B (2004) Role of an endogenous nitric oxide burst in the resistance of wheat to stripe rust. Plant Cell Environ 27: 473-477

Himanen K, Boucheron E, Vanneste S, de Almeida Engler J, Inzé D, Beeckman T (2002) Auxin-mediated cell cycle activation during early lateral root initiation. Plant Cell 14: 2339-2351

Hinchee MAW, Rost TL (1986) The control of lateral root development in cultured pea seedlings. I. The role of seedling organs and plant growth regulators. Bot Gaz 147: 137-147

Kaldorf M, Ludwig-Müller J (2000) AM fungi might affect the root morphology of maize by increasing indole-3-butyric acid biosynthesis. Physiol Plant 109: 58-67

Kojima H, Nakatsubo N, Kikuchi K, Urano Y, Higutchi T (1998) Direct evidence of NO production in rat hippocampus and cortex using a new fluorescent indicator: DAF-2DA. NeuroReport 9: 3345-3348

Kolbert Zs, Bartha B, Erdei L (2005) Generation of nitric oxide in roots of Pisum sativum, Triticum aestivum and Petroselinum crispum plants under osmotic and drought stress. Acta Biol Szeged 49: 13-16

Kolbert Zs, Bartha B, Erdei L (2008) Exogenous auxininduced $\mathrm{NO}$ synthesis is nitrate reductase-associated in Arabidopsis thaliana root primordia. J Plant Phys, in press

Lamattina L, García-Mata C, Graziano M, Pagnussat G (2003) Nitric oxide: the versatility of an extensive signal molecule. Annu Rev Plant Biol 54: 109-136

Ludwig-Müller J, Schubert B, Pieper K (1995) Regulation of IBA synthetase from maize (Zea mays L.) by drought stress and ABA. J Exp Bot 46: 423-432

Ludwig-Müller J, Vertocnik A, Town CD (2005) Analysis of indole-3-butyric acid-induced adventitious root formation on Arabidopsis root segments. J Exp Bot 56: 2095-2105
Lynch J (1995) Root architecture and plant productivity. Plant Physiol 109: 7-13

Malamy JE (2005) Intrinsic and environmental response pathways that regulate root system architecture. Plant Cell Environ 28: 67-77

Malamy JE, Benfey PN (1997) Down and out in Arabidopsis: the formation of lateral roots. Trends Plant Sci 2: 390-396

Malamy JE, Ryan KS (2001) Environmental regulation of lateral root initiation in Arabidopsis. Plant Physiol 127: 899-909

Ötvös K, Pasternak TP, Miskolczi P, Domoki M, Dorgjotov D, Szücs A, Bottka S, Dudits D, Fehér A (2005) Nitric oxide is required for, and promotes auxin-mediated activation of, cell division and embryogenic cell formation but does not influence cell cycle progression in alfalfa cell cultures. Plant J 43: 849-860

Pagnussat GC, Simontacchi M, Puntarulo S, Lamattina L (2002) Nitric oxide is required for root organogenesis. Plant Physiol 129: 954-956

Pagnussat GC, Lanteri ML, Lamattina L (2003) Nitric oxide and cyclic GMP are messengers in the indole acetic acid-induced adventitious rooting process. Plant Physiol 132: 1241-1248

Pasternak TP, Potters G, Caubergs R, Jansen MAK (2005) Complementary interactions between oxidative stress and auxins control plant growth responses at plant, organ and cellular level. J Exp Bot 56: 1991-2001

Pedroso MC, Magalhaes JR, Durzan D (2000) A nitric oxide burst precedes apoptosis in angiosperm and gymnosperm callus cells and foliar tissues. J Exp Bot 51: 1027-1036

Potters G, Pasternak TP, Guisez Y, Palme KJ, Jansen MAK (2007) Stress-induced morphogenetic responses: growing out of trouble? Trends Plant Sci 12: 98-105

Poupart J, Waddell CS (2000) The rib1 mutant is resistant to indole-3-butyric acid, an endogenous auxin in Arabidopsis. Plant Physiol 124: 1739-1751

Rashotte AM, Poupart J, Waddell CS, Muday GK (2003) Transport of the two natural auxins, indole-3-butyric acid and indole-3-acetic acid, in Arabidopsis. Plant Physiol 133: 761-772

Schneider EA, Kazakoff CW, Wightman F (1985) Gas chromatography-mass spectrometry evidence for several endogenous auxins in pea seedling organs. Planta 165: 232-241

Swarup R, Friml J, Marchant A, Ljung K, Sandberg G, Palme K, Bennett M (2007) Localization of the auxin permease AUX1 suggests two functionally distinct hormone transport pathways operate in the Arabidopsis root apex. Genes Dev 15: 2648-2653

Torrey JG (1950) The induction of lateral roots by indoleacetic acid and root decapitation. Am J Bot 37: 257-264

van der Weele CM, Spollen WG, Sharp RE, Baskin TI (2000) Growth of Arabidopsis thaliana seedlings under water 
deficit studied by control of water potential in nutrient-agar media. J Exp Bot 51: 1555-1562

Wyn Jones RG, Gorham J (1983) Osmoregulation. In: Lange OL, Nobel PS, Osmond CB, Ziegler H (eds) Encyclopedia of Plant Physiology.New Series, Vol. 12C. Springer-Verlag, Berlin, pp 35-58

Xu M, Dong J, Zhu M (2006) Nitric oxide mediates the fungal elicitor-induced puerarin biosynthesis in Pueraria thomsonii Benth. suspension cells through a salicylic acid (SA)-dependent and a jasmonic acid (JA)-dependent signal pathway. Sci China C Life Sci 49: 379-389
Zhang H, Jennings A, Barlow PW, Forde BG (1999) Dual pathways for regulation of root branching by nitrate. Proc Natl Acad Sci USA 96: 6529-6534

Zhao Z, Chen G, Zhang C (2001) Interaction between reactive oxygen species and nitric oxide in drought-induced abscisic acid synthesis in root tips of wheat seedlings. Austr J Plant Phys 28: 1055-1061

Zolman B, Yoder A, Bartel B (2000) Genetic analysis of indole-3-butyric acid responses in Arabidopsis thaliana reveals four mutant classes. Genetics 156 : 1323-1337 